\title{
The use of sexual activity measurements to assess ejaculatory performance of boars
}

\author{
Stanisław Kondracki, Maria Iwanina, Anna Wysokińska and Krzysztof Górski \\ Department of Animal Reproduction and Hygiene, Siedlce University of Natural Sciences and Humanities, Siedlce, \\ Poland
}

\begin{abstract}
The study involved 50 insemination boars at the age of 8-9 months at the beginning of the study. Each boar was assessed four times for its sexual activity: at the beginning of breeding service and after three, six and nine months into the insemination service. Ejaculates were collected for the purpose of the sexual activity parameters' assessment. Physical parameters of the ejaculates were subsequently analysed. Pearson's linear correlation was used to calculate the coefficients of a phenotypic correlation between sexual activity levels and physical parameters of the ejaculates. According to this an appropriate analysis was conducted concerning the changes in boars' sexual activity throughout their dynamic, sexual development. It was proved, that ejaculate volume depends on the total time of copulation. The highest correlation between ejaculate volume and sexual urge levels was observed in the youngest boars at the beginning of insemination service. The correlation between the ejaculatory efficiency and a boar's libido changes with age and sexual development. Changes in ejaculatory efficiency are the most dynamic at the age of approx. 1.5 years. At this age ejaculate volume and ejaculate sperm count dynamically increase. The insemination fitness forecasting in case of boars should include libido measurements taken at the beginning of an insemination service - within the first three months. Measurements taken at a more advanced age are of little use for the purpose of ejaculatory efficiency forecasting in case of boars.
\end{abstract}

Keywords: boar, ejaculate, sexual activity

Archiv Tierzucht 56 (2013) 106, 1052-1059

doi: 10.7482/0003-9438-56-106

Corresponding author:

Stanisław Kondracki; email: sk@uph.edu.pl

Department of Animal Reproduction and Hygiene, Siedlce University of Natural Sciences and Humanities, 14 Prusa Street, 08-110 Siedlce, Poland

(c) 2013 by the authors; licensee Leibniz Institute for Farm Animal Biology (FBN), Dummerstorf, Germany. This is an Open Access article distributed under the terms and conditions of the Creative Commons Attribution 3.0 License (http://creativecommons.org/licenses/by/3.0/).
Received: 14 August 2013 Accepted: 20 November 2013 Online: 2 December 2013 


\section{Introduction}

Artificial insemination currently plays a very important role in pig reproduction. The common practice of insemination in pig reproduction has contributed to a development of research on boars' insemination efficiency. On top of the economically important features, sires should have high reproductive parameters, such as: sexual activity and ejaculatory efficiency. These variables are genetically determined and depend on a breed, cross-breeding variant or genetic line, as well as environmental factors (Petherick 2005, Galina et al. 2007). Boars of different breeds display a varied sexual activity and produce ejaculates that differ in quantitative and qualitative traits (Ciereszko et al. 2000, Wolf \& Smital 2009, Kondracki et al. 2012, Wysokińska \& Kondracki 2013).

A serious problem connected with the use of boars for the insemination purposes is variability in sexual urge levels. The level of sexual excitement prior to yielding semen can affect the ejaculatory efficiency of a boar (Szostak \& Sarzyńska 2011). A stimulation of a male sexual behaviour can also influence semen quality (Pound et al. 2002, Levis \& Reicks 2005). Ejaculatory efficiency increases owing to facilitated transport of spermatozoa through the outlet ducts. There are boars that produce large amounts of high-quality semen and, simultaneously, can develop sexual reflexes quickly and easily. Such animals are particularly useful for the insemination service purposes (Bertani et al. 2002, Foote 2003, Najjar et al. 2010). In practise, insemination boars are selected chiefly according to their breeding value and market demand on sires of a particular breed or cross-breeding type. Additionally, insemination centres make sure that the acquired boars are not related to the boars previously used at a given centre. Little is known, though, about the fitness of a boar for insemination service at the moment of its acquisition. Young insemination boars should be trained to leap on the phantom while still being at the piggery where they had been raised. Their sexual urge levels are, however, largely unknown. It remains speculative, whether the intensity of copulatory reflexes' development is clearly correlated with ejaculatory efficiency, both at the beginning of service and later in the study. The current state of knowledge concerning the effect, which the sexual behaviour of boars has on their ejaculatory efficiency, is far more deficient than the knowledge of semen production physiology (Levis \& Reicks 2005). The lack of a preliminary assessment of boars' insemination fitness leads to a premature rejection or a low performance assessment. The decision to declare a boar unfit for insemination and its replacement is only taken after identifying negative results in sow's insemination. This leads not only to substantial economic losses for the users, but to even greater, though often underestimated and rarely evaluated, losses in breeding and production herds whose owners take advantage of insemination services. Sexual urge parameters can be estimated in very young boars. Some of the parameters can also prove useful in forecasting of the insemination fitness at a later stage.

The purpose of this dissertation is to test the possibilities of using sexual urge measurements in evaluation of ejaculatory efficiency of boars.

\section{Material and methods}

The study involved 50 insemination boars, which included 30 pure-breeds: Landrace and Large White, and 20 mixed, two-breeds: Duroc and Pietrain, at the age of 8-9 months at 
the beginning of the study. Each boar was tested for its sexual activity. Sexual activity measurements were examined four times in three-month intervals:

- investigation 1; in the first week of the insemination service (at the age of approx. 8-9 months),

- investigation 2; three months after the start of the insemination service (at the age of approx. 11-12 months),

- investigation 3; six months after the start of the insemination service (at the age of approx. 14-15 months),

- investigation 4; nine months after the start of the insemination service (at the age of approx. 17-18 months).

The sexual activity of the boars was assessed on the basis of the time necessary to develop successive copulatory reflexes during ejaculate collection. The following sexual activity parameters were taken into consideration:

- time from entering the arena until mounting the phantom,

- time from mounting the phantom until developing erection,

- time from entering the arena until developing erection,

- time from developing a full erection until the start of ejaculation,

- time from entering the arena until the start of ejaculation,

- duration of ejaculation,

- duration of copulation, i.e. the overall time from effectively mounting the phantom until dismounting it, following a finished ejaculation,

- number of times a boar mounted the phantom to yield semen.

The time of developing the particular sexual reflexes was determined using a stop-watch, exact to one second.

Simultaneously with testing the sexual activity parameters, we collected ejaculates for analyses. The ejaculates were manually collected into germ-free plastic bags placed in heatinsulating containers sealed with disposable viscose filters. This made it possible to directly separate the gelatinous fraction. Immediately after collection, the ejaculates were analysed for the following physical parameters: ejaculate volume, sperm concentration, percentage of spermatozoa with correct motility, total number of spermatozoa per ejaculate, and the number of insemination doses obtained from a single ejaculate. Sperm concentration in the ejaculates was determined using a photometric method through the application of a spectrophotometer. The percentage of appropriately mobile spermatozoa was identified microscopically. A light microscope with a heated stage was used for the analyses. At a 200fold zoom, we have determined the percentage of correctly mobile spermatozoa in the overall number of spermatozoa present in the vision field. The overall number of mobile spermatozoa and the number of insemination doses obtained from one ejaculate were determined using the SYSTEM SUL software v. 6.35 (Gogosystem, Poland).

The data was analysed according to the criterion of a sexual activity. To this end, the coefficients of a phenotypic correlation, which define the level of correlation between the physical ejaculate parameters and a boar's sexual activity, were calculated at the beginning of a boar's service and after three, six and nine months into the insemination service. The correlation coefficients were calculated in reference to Pearson's linear correlation. An analysis of changes in boars' sexual activity occurring with their continuous sexual development was 
also performed. The data was analysed using the following mathematical model:

$$
Y_{i j}=\mu+a_{i}+e_{i j}
$$

where $Y_{i j}$ is the value of the analysed parameter, $\mu$ is the populational mean, $a_{i}$ is the effect of sexual development, $e_{i j}$ is the error.

Tukey's test was used to assess the significance of inter-group differences.

\section{Results and discussion}

Table 1 presents the results of the boars' sexual activity assessment at the beginning of the programme, and after three, six and nine months into the insemination service. The data indicates that the young boars both, at the beginning of the service and after three months, needed relatively little time to mount the phantom after entering the arena (180.34 and 156.30 seconds, respectively). Older boars, after six and nine months of service, needed between 45 and 102 seconds more to mount the phantom. The time from mounting the phantom to developing an erection also increased as the boars gained more and more of sexual experience. This seems to show, that the sexual experience of insemination boars is not conducive of the intensity of their sexual behaviour expression. There is no doubt that copulatory experience and sexual skills of males improve with age (Rhen \& Crews 2002). The data of the present study show, however, that these factors do not positively affect the boars' expression of their sexual behaviour. On the contrary, older boars need more time to effectively mount the phantom. At the same time, the ejaculatory efficiency clearly rises as the young, though already performing, boars develop. Changes in ejaculatory efficiency are the most dynamic at the age of approx. 1.5 years (table 2). At this age ejaculate volume and ejaculate sperm count dynamically increase. According to some authors, however, the volume of yielded ejaculates and the number of spermatozoa contained in the ejaculate, keep rising until approx. $27-28$ months of age, and only then become stabilised at a relatively steady level (Kondracki et al. 2004, Banaszewska \& Kondracki 2012). We, therefore, deal with a situation in which structures responsible for the production of male sex hormones and the triggering of a manifested libido, develop relatively early and reach the maximum development level in boars at 7-10 months of age. This is confirmed by the data presented in Table 1, which shows that young boars examined at the beginning and three months after of the start of the insemination service need relatively little time to develop copulatory reflexes. The same boars examined six and nine months into the service, require much more time to exhibit similar sexual behaviour. Simultaneously, there is a dynamic development of structures responsible for the generation of semen plasma. This development takes much longer than the development of Sertoli cells that generate sex hormones. This results in a continuous increase in ejaculate volume. This precisely seems to be the reason why the correlation between ejaculate volume and the level of a manifested libido is much higher at a younger age than later into the insemination service of the boars. It seems then, that insemination fitness forecasting in case of boars should include libido measurements taken at the beginning of the insemination service - within the first three months. Measurements taken at a more advanced age are of little use for ejaculatory efficiency forecasting in case of boars. 


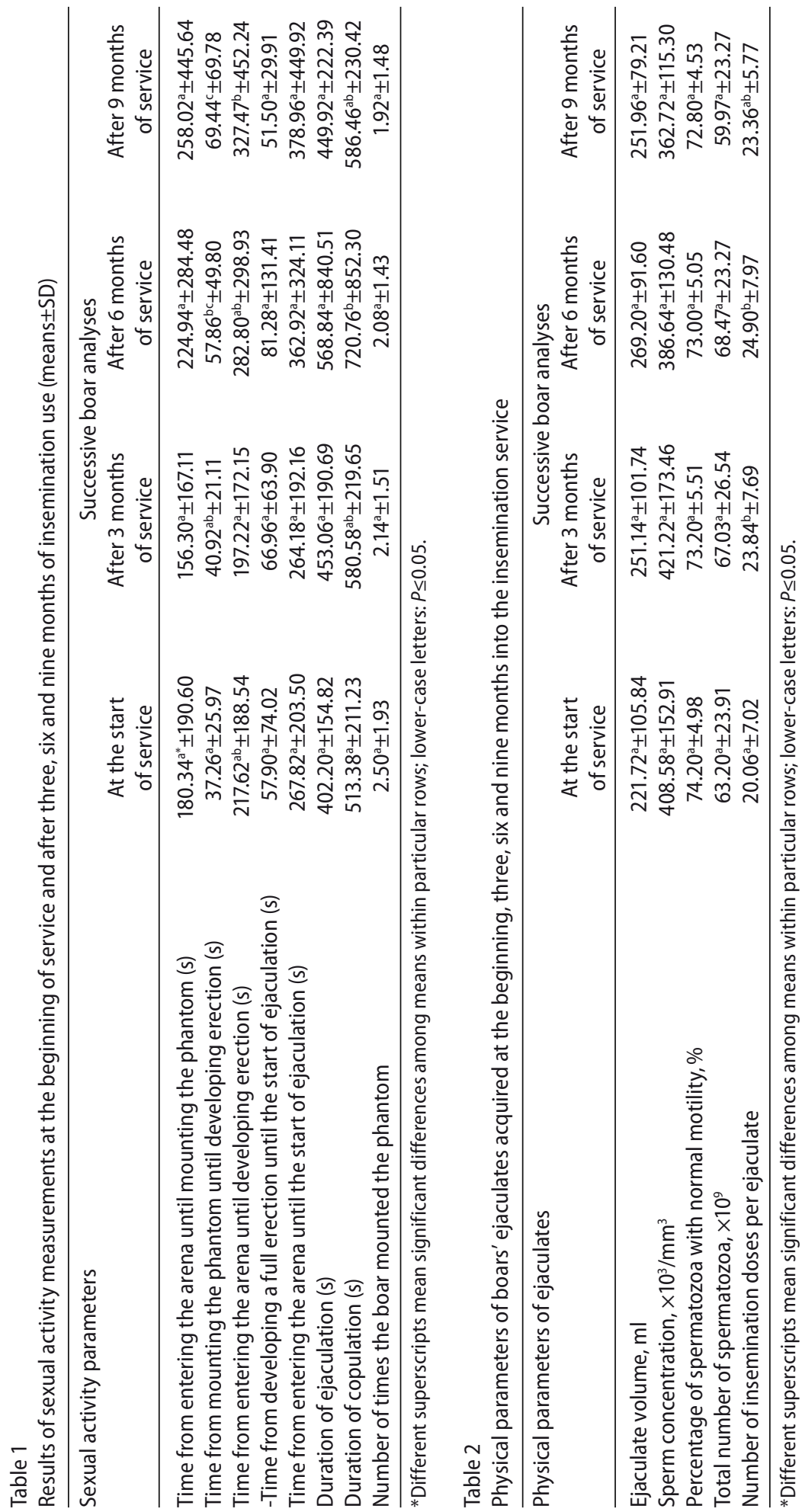


Table 3 contains a juxtaposition of data that define the interrelation between physical ejaculate parameters and a boar's sexual activity. The data show that the physical parameters of the ejaculate are loosely correlated with sexual urge levels. It was proved, however, that ejaculate volume depends on the duration of ejaculation $(r=0.28 ; P<0.05)$ and on the overall duration of copulation $(r=0.27 ; P<0.05)$. It was the ejaculation time and overall semen collection time that the physical ejaculate parameters were the most closely correlated with. On the other hand, ejaculate parameters only slightly depended on the other sexual urge characteristics.

The highest correlation between ejaculate volume and sexual urge levels was observed in the youngest boars at the beginning of the insemination service (table 4). The volume of ejaculates collected in the first week of the insemination service was highly correlated with the duration of ejaculation and the overall duration of copulation, ascertained when collecting the semen ( $r=0.66$ and $r=0.56$ for the duration of ejaculation and overall copulation time, respectively). Further into the service and sexual development of the boars, the correlation of ejaculate volume with the duration of ejaculation and the overall duration of copulation diminished. The correlation was still relatively high after three months of service, as evidenced in the high phenotypic correlation coefficients which then amounted to 0.61 for ejaculation duration, and 0.47 for the overall copulation time respectively $(P<0.05)$. In the case of older boars examined after six and nine months into the insemination service, these correlations were significantly smaller, and the coefficients of a phenotypic correlation did not exceed 0.3 ; being statistically unconfirmed $(P>0.05)$. The data proves that the correlation between the ejaculatory efficiency and a boar's libido changes with age and sexual development. Boars usually begin their insemination service at approx. 7-8 months of age. They are still very young at the time, still sexually developing, though mature and ready for copulation (Oberlender et al. 2012). Ejaculates obtained from such boars can exhibit high variability. Along with sexual development, there are changes occurring in the levels of sex hormones, particularly in testosterone, which plays a major role in maintaining spermatogenesis and an adequate sexual behaviour (Williams 2009).

The data in Table 4 show the correlation between the ejaculate volume and a boar's libido. The intensity of this dependence diminishes, however, with age and sexual development of the boar. This may be due to different rates of development of particular male reproductive system elements. Interstitial testicular cells (Leydig cells), responsible for the production of sex hormones, develop relatively early and determine the strong sexual drive in relatively young males, which grow to a lesser degree at a more advanced age (Weiler \& Claus 1991, Ellison et al. 1993, Zeng et al. 2001, Szostak \& Sarzyńska 2011). This seems to be corroborated by the data presented in Table 3, which shows that both an extended service and sexual development of boars are not accompanied at all by an increase in the expression of a sexual behaviour.

To summarise, it must be concluded that the insemination fitness assessments of boars can involve sexual urge measurements examined the beginning of a reproductive service. The measurements of an ejaculation duration and overall copulation time applied at the beginning of an insemination service are the most useful for this purpose. 


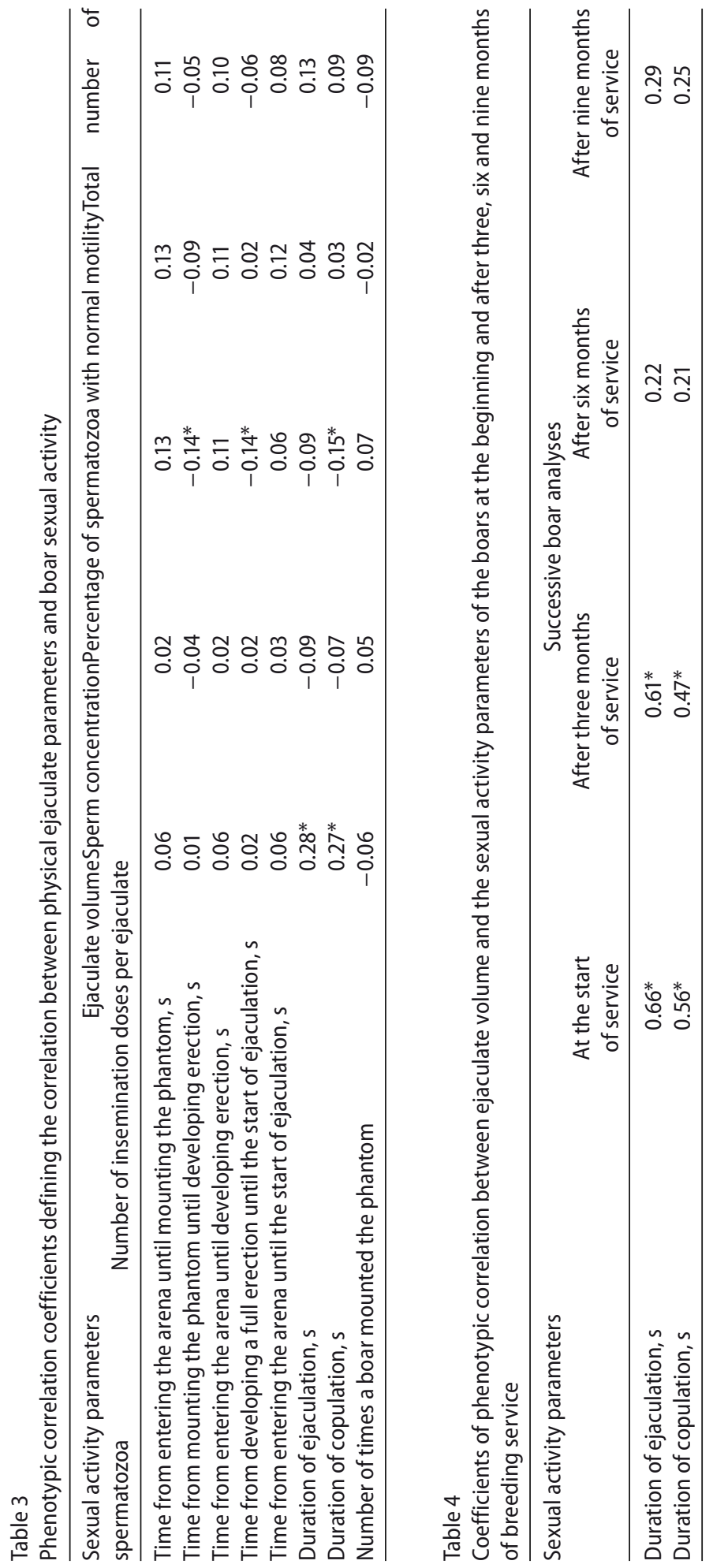




\section{Acknowledgements}

This dissertation has been prepared as part of the project granted by the National Centre for Research and Development Nr N R12 0013 06/2009.

\section{References}

Banaszewska D, Kondracki S (2012) An Assessment of the Breeding Maturity of Insemination Boars Based on Ejaculate Quality Changes. Folia Biol (Krakow) 60, 151-162

Bertani GR, Scheid IR, Irgang R, Barioni Jr W, Wentz I, Afonso SB (2002) Gonadal sperm reserve in purebred Landrace and Large White boars of high average daily gain. Theriogenology 57, 859-867

Ciereszko A, Ottobre JS, Glogowski J (2000) Effects of season and breed on sperm acrosin activity and semen quality of boars. Anim Reprod Sci 64, 89-96

Ellison PT, Panter-Brick C, Lipson SF, O'Rourke MT (1993) The ecological context of human ovarian function. Hum Reprod 8, 2248-2258

Foote RH (2003) Fertility estimation: a review of past experience and future prospects. Anim Reprod Sci 75, 119-139

Galina CS, Horn MM, Molina R (2007) Reproductive behaviour in bulls raised under tropical and subtropical conditions. Horm Behav 52, 26-31

Kondracki S, Banaszewska D, Wysokinska A, Kosieradzka J (2004) Effect of age on semen traits of young landrace boars. J Agrobiol Ecol 1 (1-2), 112-117

Kondracki S, Iwanina M, Wysokińska A, Huszno M (2012) Comparative analysis of Duroc and Pietrain boar sperm morphology. Acta Vet Brno 81, 195-199

Levis DG, Reicks DL (2005) Assessment of sexual behavior and effect of semen collection pen design and sexual stimulation of boars on behavior and sperm output-a review. Theriogenology 63, 630-642

Najjar A, Benaoun B, Ezzaouia M, Ben Maâtoug A, Magistrini M, Ben Mrad M (2010) Determination of Semen and Sexual Behavior Parameters of Arabian Stallions to Be Selected for an Artificial Insemination Program under Tunisian Conditions. American-Eurasian J Agric Environ Sci 8, 173-177

Oberlender G, Murgas LDS, Zangeronimo MG, Silva AC, Pereira LJ (2012) Influence of Ejaculation Time on Sperm Quality Parameters in High Performance Boars. J Anim Sci Adv 2, 499-509

Petherick JC (2005) A review of some factors affecting the expression of libido in beef cattle, and individual bull and herd fertility. Appl Anim Behav Sci 90, 185-205

Pound N, Javed MH, Ruberto C, Anis Shaikh M, Del Valle AP (2002) Duration of sexual arousal predicts semen parameters for masturbatory ejaculates. Physiol Behav 76, 685-689

Rhen T, Crews D (2002) Variation in Reproductive Behaviour within a Sex: Neural Systems and Endocrine Activation. J Neuroendocrinol 14, 517-531

Szostak B, Sarzyńska J (2011) The influence of the breed and age on the libido of insemination boars. Acta Sci Pol Zootec 10, 103-110

Weiler U, Claus R (1991) Endocrine aspects of testicular function, especially hormones in the seminal plasma and their fate in the female reproductive tract: testicular steroids and their relevance for male and female reproductive functions. Reprod Domest Anim 26 (Suppl.), 41-61

Williams S (2009) Assessment of the boar reproductive efficiency: physiology and implications. Rev Bras Reprod Anim 33 (Suppl.), 194-198

Wolf J, Smital J (2009) Quantification of factors affecting semen traits in artificial insemination boars from animal model analyses. J Anim Sci 87, 1620-1627

Wysokińska A, Kondracki S (2013) Assessment of the effect of heterosis on semen parameters of two-breed crosses of Duroc, Hampshire and Pietrain boars. Arch Tierz 56, 65-74

Zeng XY, Turkstra JA, Van De Wiel DFM, Guo DZ, Liu XY, Meloen RH, Schaaper WMM, Chen FQ, Oonk HB, Zhang $X$ (2001) Active Immunization against Gonadotrophin-releasing Hormone in Chinese Male Pigs. Reprod Domest Anim 36, 101-105 